\title{
A REVIEW ON BIOANALYTICAL METHOD DEVELOPMENT AND VALIDATION
}

\author{
TIJARE LK*, RANGARI NT, MAHAJAN UN
}

Department of Quality Assurance, Dadasaheb Balpande Collage of Pharmacy, (Besa) Nagpur, Maharashtra, India. Email: lokuktijare.2015@rediffmail.com

Received: 26 July 2016, Revised and Accepted: 24 August 2016

\section{ABSTRACT}

In this review article, bioanalytical methods are widely used to quantitate drugs and their metabolites in plasma matrices and the methods should be applied to studies in areas of human clinical and nonhuman study. Bioanalytical method employed for the quantitative estimation of drugs and their metabolites in biological media and plays an important role in estimation and interpretation of bioequivalence, pharmacokinetic, and toxicokinetic studies. The major bioanalytical role is method development, method validation, and sample analysis. Every step in the method must be investigated to decide the extent to which environment, matrix, or procedural variables can interfere the estimation of analyte in the matrix from the time of set up to the time of analysis. Techniques such as high pressure liquid chromatography (HPLC) and liquid chromatography coupled with double mass spectrometry (LCMS-MS) can be used for the bioanalysis of drugs in body. Each of the instruments has its own merits and demerits. Chromatographic methods are HPLC and gas chromatography have been mainly used for the bioanlysis of small/ large molecules, with LC/MS/MS. Linearity, accuracy, precision, selectivity, sensitivity, reproducibility, and stability are some of the regularly used parameters. In this review article, we are proposed to add some points regarding bioanalytical method development and validation parameter, beneficial to quality assurance to determine the drug, concentration and its metabolite.

Keywords: Method development, Clinical and nonclinical study, Analyte, Validation of bioanlysis techniques, Validation parameter.

(C) 2016 The Authors. Published by Innovare Academic Sciences Pvt Ltd. This is an open access article under the CC BY license (http://creativecommons. org/licenses/by/4. 0/) DOI: http://dx.doi.org/10.22159/ajpcr.2016.v9s3.14321

\section{INTRODUCTION}

In this methods determine the drugs in biological fluid are becoming increasingly important for the study of bioavailability, bioequivalence (BE) Pharmacokinetics (PK) studies, quantitative evaluation of drugs, concentration and their metabolites, new drug development, research in basic biomedical and pharmaceutical sciences and therapeutic drug monitoring etc., [1,2] High pressure liquid chromatography (HPLC) most widely applied analytical techniques because of its highly selective and high reliability, especially in the pharmaceutical, environmental, forensic, clinical, and food department [3].

Generally in 2001 by USFDA and recently the EMEA also proposed the guidelines for the bioanalytical method validation. Validation involves recording, through the use of specific laboratory investigations, that the performance characteristics of a method are suitable and reliable for the intended bioanalytical applications. The acceptability of analytical data corresponds to used validate the method. For important studies that require regulatory action for approval, such as BE or PK studies, the bioanalytical methods could be fully validated. For advance methods used for the sponsor's inner decision-making, less validation may be sufficient when changes are made to a previously validated method, additional validation may be needed $[4,5]$. Often undergoes many modifications; these modifications should be validated to confirm suitable performance of the analytical method. The evolutionary changes needed to support specific studies for the different levels of validation to demonstrate the validity of method Validation of bioanalytical methods carried during:

- During development and implementation of a novel bioanalytical method.

- For analysis of a new drug entity.

- For revisions to an existing method that add metabolite quantification [6].

- Bioanalytical method transfers between laboratories or analysts.

- Change in analytical methodology.

- Change in matrix within species (e.g., human plasma to human urine).

- Change in sample processing procedures [7].

\section{METHOD DEVELOPMENT}

Bioanalytical method development is the process of making a procedure to unknown compound or novel compound be identified and measured in a matrix. A compound can often be measured by several methods and the choice of analytical method involves, that is, chemical properties of the analyte, concentrations, sample matrix, cost of the analysis method and instruments, speed and time of the analysis, quantitative or qualitative measurement, precision and necessary equipment. Method development includes sample preparation sampling, separation, detection and evaluation of the results and finally conclusion [8].

\section{Sample collection and preparation}

The living media that contain the analyte are usually blood, plasma, urine, serum, etc. Blood is usually collected from human volunteers/ subjects by vein puncture with a hypodermic syringe up to 5-7 $\mathrm{ml}$. The venous blood is withdrawn into tubes with an anticoagulant, generally ethylenediaminetetraacetic acid, heparin is used. Plasma is obtained by centrifugation at $4000 \mathrm{rpm}$ for 15 minutes. Around $30-50 \%$ of the volume is collected. The aim of sample preparation is to clean up the sample before analysis. Material in biological samples that can affect with analysis, the chromatographic column or the detector includes endogenous macromolecules, proteins, salts, small molecules, and metabolic by products. The sample preparation is also to conversation the analyte from the biological matrix into a solvent suitable for instillation into the chromatographic system. General methods for sample preparation such as liquid/liquid extraction, solid-phase extraction (SPE) and protein precipitation, chromatography, and ligand binding assay (LBA) $[9,10]$.

\section{BIOANALYTICAL METHOD}

Some of the following bioanalytical method:

- Extraction method

- Protein precipitation 
- Chromatography method

- Ligand binding assay (LBA).

\section{Extraction method}

\section{Liquid-liquid extraction}

It is based on the principles of difference solubility and partitioning equilibrium of analyte molecules between aqueous (the sample) and the organic phases. Liquid-liquid extraction generally involves the extraction of a substance from one liquid phase to additional liquid phase [11]. Nowadays liquid extraction replaced with advanced and improved methods like liquid phase micro extraction and supported membrane extraction, single drop liquid phase micro extraction [12].

\section{$S P E$}

SPE is choosy method for sample preparation where the analyte is bound onto a solid support, interferences are washed off and the analyte is selectively eluted. No. of choices of sorbents, SPE is a very powerful technique. Solid phase includes four steps; conditioning, sample loading, washing and elution are shown in Fig. 1.

I. Conditioning

The column is triggered with an organic solvent that acts as a wetting agent on the packing material and solvates the functional groups of the sorbent. Water or aqueous buffer is added to activate the column for proper adsorption mechanisms.

II. Sample loading

After adjustment of $\mathrm{pH}$, the sample is entering on the column by gravity feed, pumping or aspirating by vacuum.

III. Washing

Interferences from the matrix are removed while retaining the analyte.

IV. Elution

Distribution of analyte - sorbent interactions by suitable solvent, removing as little of the remaining interferences as possible. Generally, sorbents used in SPE consists of $40 \mu \mathrm{m}$ diameter silica gel with around $60 \mathrm{~A}^{0}$ pore diameters. To this silica gel, functional groups are chemically bonded. The most commonly used format is a syringe barrel that contains a $20 \mu \mathrm{m}$ frit at the bottom of the syringe with the sorbent material and another frit on top, referred to as packed columns. Extractions disks are placed in syringe barrels. These disks consist of 8-12 $\mu \mathrm{m}$ particles of packing material fixed into an inert matrix. Disks are conditioned and used in a similar way as packed columns. The major advantage of disks compared to packed columns is that higher flow rates can be easily applied. Analytes can be classified into four categories; acid, basic, neutral, and amphoteric compounds. Amphoteric analytes have both acid and basic functional groups and can, therefore, functions as cations, anions or zwitterions, depending on $\mathrm{pH}$, mainly the $\mathrm{pH}$ is 13 [13-15].

\section{Protein precipitation}

Protein precipitation is widely used in routinely analysis to remove proteins. Precipitation can be induced by the addition of an organic

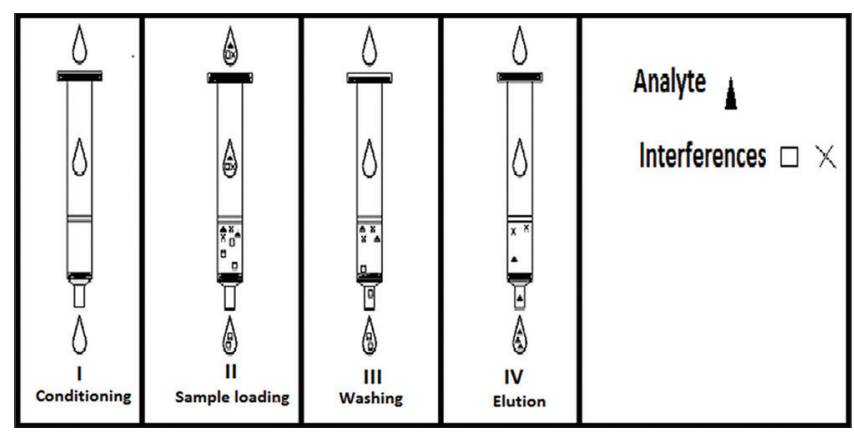

Fig. 1: Steps in solid phase extraction modernizer, a salt or by changing the ph which influence the solubility of the proteins. The samples are centrifuged and the supernatant can be inserted into the HPLC system or be evaporated to dryness and dissolved in a suitable solvent. A concentration of the sample is then achieved. There are some aids with precipitation method as cleanup technique compared to SPE [16]. It is less time-consuming, little amounts of organic modifier or other solvents are used. But there are also disadvantages; the samples often contain protein particles and it is a no-selective sample cleanup method, there is a risk that endogenous compounds or other drugs may restrict in the reversed phaseHPLC - system. However, the protein precipitation technique is often combined with SPE to produce clean extract. Methanol is generally favored solvent among the organic solvents as it can produce clear supernatant which is appropriate for direct addition into HPLC. Salts are other alternative to acid organic solvent precipitation. This technique is called as salt induced precipitation. As the salt concentration of a solution is increased, proteins aggregate and precipitate from the solution $[17,18]$

\section{Chromatographic method}

\section{Reference standards}

Analysis of drugs and their metabolites in biological fluids is performed using calibration Standards and quality control samples (QCs) spiked with reference standards. The purity of the reference standard used to prepare spiked samples can affect study data. For this reason, Authenticated analytical reference standards of known identity and purity must be used to prepare solutions of known concentrations. If possible, the reference standard should be identical to the analyte. When this is not possible, a predictable chemical form (free base or acid, salt or ester) of known purity can be used [12].

Three types of reference standards are usually used:

- Certified reference standards (e.g., USP compendial standards).

- Commercially-supplied reference standards obtained from a reputable commercial source.

- Other materials of documented purity custom-synthesized by an analytical laboratory or other noncommercial establishment.

The source, expiration date, lot number, documentations of analyses when existing, and/or internally or externally generated evidence of identity and purity should be unfurnished for each reference and internal standard (IS) used. If the reference or IS expired, stock solutions made with this lot of standard should not be used unless purity is reestablished $[19,20]$.

\section{LBA}

Numerous of the bioanalytical validation parameters and principles discussed above are also applicable to microbiological and LBA. These types of assays have a variety of design configurations that possess some unique features that should be considered during method validation.

\section{Key reagents}

Key reagents, such as reference standards, antibodies, tracers, and matrices should be characterized appropriately and stored under defined conditions. Assay reoptimization or validation may be important when there are changes in key reagents.

For example:

Labeled analytes (tracers): Binding should be reoptimized and Performance should be verified with standard curve and QCs.

Antibodies: Key cross-reactivates should be checked. Tracer experiments above should be repeated.

Matrices: Tracer experiments above should be repeated [18]. 


\section{BIOANALYTICAL METHOD VALIDATION}

\section{Need of bioanalytical method validation}

- It is essential to used well-characterized and fully validated bioanalytical methods to yield reliable results that can be satisfactory interpreted.

- It is recognized that bioanalytical methods and techniques are constantly undergoing changes and improvements; they are at the cutting edge of the technology.

- It is also important to emphasize that each bioanalytical technique has its own characteristics, which will vary from analyte to analyte, specific validation criteria ma need to be developed for each analyte.

- Moreover, the appropriateness of the technique may also be influenced by the ultimate objective of the study. When samples analysis for a given study is conducted at more than one site, it is necessary to validate the bioanalytical methods at each site and provide appropriate validation information for different sites to establish inter-laboratory reliability [21].

\section{Linearity and range}

A calibration curve is the relationship between response and known concentration of the analyte. The calibration curve should be prepared in the same biological matrix as the samples and a calibration curve should be generated for each analyte. The range of the method is the concentration interval where accuracy, precision, and linearity have been validated. The used calibration curve should be the simplest model that adequately describes the concentration-response relationship. The deviation should not exceed more than $20 \%$ from the nominal concentration of the lower limit of quantification (LLOQ) and not more than $15 \%$ from the other standards in the curve.

\section{Accuracy}

The accuracy of an analytical method describes the closeness of test results obtained by the method to the true value of the analyte. Accuracy is determined by replicate analysis of samples containing known amounts of the analyte. Accuracy should be measured using a minimum of five determinations per concentration. A minimum of three concentrations in the range of expected. The mean value should be within $15 \%$ of the actual value except at LLOQ where it should not deviate by more than $20 \%$. The deviation of the mean from the true value serves as the measure of accuracy [22,23].

\section{Bias}

According to ISO, bias is the difference between the expectation of test results and an accepted reference value. It may consist of more than one systematic error component. Bias can be measured as a percent deviation from the accepted reference value. The term trueness expresses the deviation of the mean value of a large series of measurements from the accepted reference value. It can be expressed in terms of bias. Due to the high workload of analyzing such large series, trueness is usually not determined during method validation, but rather from the results of a great number of QCs during routine application [24].

\section{Precision}

The precision of an analytical method describes the closeness of individual measures of an analyte when the procedure is applied repeatedly to multiple aliquots of a single homogeneous volume of biological matrix. Precision should be measured using a minimum of five determinations per concentration. A minimum of three concentrations in the range of expected concentrations is needed. The precision determined at each concentration level should not exceed $15 \%$ of the coefficient of variation (CV) except for the LLOQ, where it should not exceed $20 \%$ of the CV. Precision is further subdivided into inter day, intraday and different analyst or repeatability, this carried out precision or repeatability measure, which measures precision with time and may involve different analysts, equipment, reagents and laboratories [25].

\section{Intermediate precision}

Intermediate precision expresses within-laboratories variations: Different days, different analysts, different equipment's, etc. [17] The
ISO definition used the term "M-factor different intermediate precision," where the M-factor expresses the number of factors (operator, equipment, or time) that differ between successive determinations. Intermediate precision is sometimes also called between-run, betweenday, or inter-assay precision [8].

\section{Selectivity}

Selectivity exercise is carried out to assess the ability of the bioanalytical method to differentiate and quantify the analyte in the presence of other components in the sample. For selectivity, analyses of blank samples of appropriate biological matrix (plasma, urine, or other matrix) obtained from at least six sources should be carried out. Each blank sample should be tested for interference and selectivity should be ensured at the lower LOQ (LLOQ) [7].

\section{Limit of detection (LOD)}

The LOD is a characteristic for the limit test only. It is the lowest amount of analyte in a sample that can be detected but not necessarily quantified under the stated experimental conditions. The detection is usually expressed as a percentage, parts per million, or parts per billion.

\section{LOQ}

LLOQ is the small amount of analyte present in a sample that can be determined quantitatively with suitable accuracy and precision. Determining LLOQ on the basis of accuracy and precision is probably the most practical method and defines the LLOQ as the lowest concentration of the sample that can still be quantified with acceptable accuracy and precision. LLOQ based on signal and noise ratio can only be applied only if baseline noise, for example chromatographic methods [26].

\section{Recovery}

The recovery of an analyte assay is the response of detector obtained from an amount of the analyte added to and extracted from the biological fluids, compared to the detector response obtained from the true concentration of the pure authentic standard. Recovery of the analyte is not necessary to be $100 \%$, but they are extent to recovery of an analyte and of the IS should be precise, consistent, and repeated. Recovery experiments should be performed by comparing the obtained results for extracted samples at three least concentrations (low, medium, and high) with un extracted standards that represent $100 \%$ recovery [2].

\section{Robustness}

According to the ICH guidelines, the robustness of an analytical procedure is the measure of its capacity to remain unaffected by small, but deliberate variations in method parameters and provides an indication of its reliability during normal usage. Robustness can be described as the ability to reproduce the analytical or bioanalytical method in different laboratories or under different environment without the occurrence of unexpected differences in the obtained result.

\section{Ruggedness}

Ruggedness is a measure for the susceptibility of a method to small changes that might occur during routine analysis like small changes of $\mathrm{pH}$ values, mobile phase composition, temperature, etc. Full validation must not necessarily include ruggedness testing; it can, however, be very helpful during the method development/prevalidation phase, as problems that may occur during validation are often detected in advance. Ruggedness should be tested if a method is supposed to be transferred to another laboratory $[27,28]$.

\section{Stability}

The stability of the analyte under various conditions should also be studied during method validation. The conditions used in stability experiments should reflect situations likely to be encountered during actual sample handling and analysis. The following stability conditions are stated by FDA and are advisable to investigate [29]; 


\section{Stock solution stability}

The stability of the stock solution must be evaluated at room temperature for the $6 \mathrm{hrs}$.

\section{Short-term temperature stability}

The stability of the analyte in biological fluids at ambient temperature should be evaluated. Three aliquots of low and high concentration kept for at least $24 \mathrm{hrs}$ and then analyzed.

\section{Long-term temperature stability}

The stability of the analyte in the matrix should beyond the time from sample collection until the last day of analysis.

\section{Freeze and thaw stability}

The stability of the analyte should be determined, after three freeze and thaw cycles. Three aliquots of low and high concentration should be icy for $24 \mathrm{hrs}$ and then thawed at room temperature.

\section{Post-preparative stability}

The stability of the analyte during stages of process of analysis should be evaluated $[30,31]$

\section{Application of validated method for routine drug analysis}

Assays of all samples of an analyte in a biological matrix should be completed within the time period for which stability information are available. In general, biological samples can be analyzed with a single determination without duplicate or replicate analysis if the assay method has satisfactory acceptable variability as defined by validation data [32]. This is true for the procedures where accuracy and precision and variabilities routinely fall within acceptance limits. For a not easy procedure with a labile analyte where high precision and accuracy specifications may be difficult to achieve, duplicate, or even triplicate analyses can be performed for a better estimation of analyte.

The following recommendations should be noted in applying a bioanalytical method to routine drug analysis.

- A matrix-based standard curve should consist of a minimum of six to nine standard points, excluding blanks (either single or duplicate), covering the entire range.

- Response function: Typically, the same curve fitting, weighting, and goodness of fit determined during pre-study validation would be used for the standard curve within the study. Response function is determined by appropriate statistical tests based on the actual standard points during every run in the validation. Changes in the response function relationship between pre-study validation and routine run validation indicate number of problems [33].

- The QC samples must be used to accept or reject the run. These QC samples are matrix spiked with analyte [24].

- System suitability: Based on the analyte and technique, a specific standard operating procedure (or sample) must be identified to ensure optimum operation of the system used.

- Any required sample dilutions should use like matrix (e.g., human to human) obviating the need to incorporate actual within-study dilution matrix in QC samples.

- Repeat analysis: It is important to establish an SOP or guideline for repeat analysis and acceptance criteria. This SOP or guideline explains the reasons for repeating sample analysis. Reasons for repeat analyses might be include repeat analysis of clinical or preclinical samples for the use of regulatory purposes, inconsistent replicate analysis, samples outside of the assay limit, sample processing errors, equipment failure, poor chromatography, and inconsistent PK data. Reassay must be done in triplicate if the sample volume allows. The basis for the repeat analysis and the reporting of the repeat analysis should be clearly documented.

- Sample data reintegration: An SOP or guideline for sample data reintegration should be established. This SOP or guideline should explain the reasons for reintegration and how the reintegration is to be performed. The rationale for the reintegration should be clearly described and documented. Original and reintegration data should be reported [34].

\section{CONCLUSION}

Bioanalysis and the production of pharmacokinetic, toxicokinetic, and metabolic data plays a fundamental role in pharmaceutical research, development involved in the drug discovery and development process. An attempt has been made to understand and explain the bioanalytical method development and validation from a quality assurance department point view. Some of the method and how is validation carried out were described in different situations encountered in the study sample analysis has been reported in this article. These various essential development and validation characteristics for bioanalytical methodology have been discussed with a view to improving the standard and acceptance in this area of research.

\section{REFERENCES}

1. Thompson M, Ellison SL, Wood R. Harmonized guidelines for single laboratory validation of method of analysis. Pure Appl Chem 2008;74(5):835-55.

2. Wood R. How to validate analytical methods. Trends Analyt Chem 2005;18:624-32.

3. Chiu ML, Lawi W, Snyder ST, Wong PK, Liao JC, Gau V. Matrix effects: A challenge toward automation of molecular analysis. J Assoc Lab Autom 2010;15:233-42.

4. Reid E, Wilson ID. Methodological survey in biochemistry and analysis. Analysis for Drug and Metabolites, Including Anti-Infective Agents. Vol. 20. Cambridge, England: Royal Society of Chemistry; 1990. p. $1-57$.

5. Surendra B, DeStefano A. Key elements of bioanalytical method validation for small molecules. AAPS J 2007;9(1):109-14.

6. McDowall RD. The role of laboratory information management systems LIMS in analytical method validation. Anal Chim Acta 2007;54:149-58.

7. Vander Heyden Y, Nijhuis A, Smeyers-Verbeke J, Vandeginste BG, Massart DL. Guidance for robustness/ruggedness tests in method validation. J Pharm Biomed Anal 2001;24(5-6):723-53.

8. Puluido A, Ruusanches I, Boque R, Rius FX. Uncertainty of results in routine qualitative analysis in analytical chemistry. J Pharm Biomed Anal 2005;22:647-54

9. Kallner A. Quality specifications based on the uncertainty of measurement. Scand J Clin Lab Invest 1999;59(7):513-6.

10. Rao KR, Kumar KS. Bioanalytical method validation-A quality assurance auditor view point. J Pharm Sci Res 2009;1(3):1-10.

11. Lang JR, Bolton S. A comprehensive method validation strategy for bioanalytical applications in the pharmaceutical industry--1. Experimental considerations. J Pharm Biomed Anal 1991;9(5):357-61.

12. Causon R. Validation of chromatographic methods in biomedical analysis. Viewpoint and discussion. J Chromatogr B Biomed Sci Appl 1997;689(1): 175-80.

13. Shah VP. The history of bioanalytical method validation and regulation: Evolution of a guidance document on bioanalytical method validation. AAPS J 2007;9(1):43-7

14. Buick AR, Doig MV, Jeal SC, Land GS, McDowall RD. Method validation in the bioanalytical laboratory. J Pharm Biomed Anal 1990;8(8-12):629-37.

15. Tiwari G, Tiwari R. Bioanalytical method validation: An updated review. Pharm Methods 2010;1(1):25-38.

16. Jhang JS, Chang CC, Fink DJ, Kroll MH. Evaluation of linearity in the clinical laboratory. Arch Pathol Lab Med 2004;128(1):44-8.

17. Mark H. Application of improved procedure for testing linearity of analytical method to pharmaceutical analsysis. J Pharm Biomed Anal 2003;33(1):7-20.

18. Hartmann C, Smeyers-Verbeke J, Massart DL, McDowall RD. Validation of bioanalytical chromatographic methods. J Pharm Biomed Anal 1998;17(2):193-218

19. Food and Drug Administration. Guidance for Industry: Bioanalytical Method Validation. Vol. 13. Rockville, MD: U.S Department of Health and Human Services, Food and Drug Administration; 2001. p. 385-94.

20. Wells DA. High throughput bioanalytical sample preparation: Methods and automation strategies. Progress in Pharmaceutical and Biomedical Analysis. Vol. 30. Amsterdam, London: Elsevier; 2003. p. 610. 
21. Karnes HT, Shiu G, Shah VP. Validation of bioanalytical methods Pharm Res 1991;8(4):421-6.

22. Kringle RO. An assessment of the 4-6-20 rule of acceptance of analytical runs in bioavailability, bioequivalence, and pharmacokinetic studies. Pharm Res 1994;11(4):556-60.

23. Wieling J, Hendriks G, Tamminga WJ, Hempenius J, Mensink CK, Oosterhuis B, et al. Rational experimental design for bioanalytica methods validation. Illustration using an assay method for total captopril in plasma. J Chromatogr A 1996;730(1-2):381-94.

24. Kringle R, Hoffman D. Stability methods for assessing stability of compounds in whole blood for clinical bioanalysis. Drug Inf $\mathrm{J}$ 2001;35:1261-70.

25. Viswanathan CT, Bansal S, Booth B, DeStefano AJ, Rose MJ, Sailstad J, et al. Quantitative bioanalytical methods validation and implementation: Best practices for chromatographic and ligand binding assays. AAPS J 2007;9(2):E260-7.

26. Singh PS, Shah G. Analytical method development and validation. J Pharm Res 2011;4(5):2330-2.

27. Dadgar D, Burnett PE. Issues in evaluation of bioanalytical method selectivity and drug stability. J Pharm Biomed Anal 1995;14(1-2):23-31.
28. Miller KJ, Bowsher RR, Celniker A, Gibbons J, Gupta S, Lee JW, et al. Workshop on bioanalytical methods validation for macromolecules: Summary report. Pharm Res 2001;18(9):1373-83.

29. Hubert H, Chiap P, Crommen J, Boulanger B, Chapuzet E, Mercier N, et al. The SFSTP guide on the validation of chromatographic methods for drug analysis: From the Washington Conference to the laboratory. Anal Chim Acta 1999;391:45-55.

30. Timm U, Wall M, Dell D. A new approach for dealing with the stability of drugs in biological fluids. J Pharm Sci 1985;74(9):972-7.

31. Nowatzke W, Woolf E. Best practices during bioanalytical method validation for the characterization of assay reagents and the evaluation of analyte stability in assay standards, quality controls, and study samples. AAPS J 2007;9(2):E117-22.

32. Braggio S, Barnaby RJ, Grossi P, Cugola M. A strategy for validation of bioanalytical methods. J Pharm Biomed Anal 1996;14(4):375-88.

33. James CA, Breda M, Frigerio E. Bioanalytical method validation: Arisk-based approach? J Pharm Biomed Anal 2004;35(4):887-93.

34. Boulanger B, Chiap P, Dewe W, Crommen J, Hubert P. An analysis of the SFSTP guide on validation of chromatographic bioanalytical methods: Progresses and limitations. J Pharm Biomed Anal 2005;32(4-5):753-65. 